[CONTRIBUTION FROM THE Chemical, LABORATORY OF ThE UNIVERSITY OF ILIINOIS]

\title{
THE USE OF THE OXIDES OF PLATINUM FOR THE CATALYTIC REDUCTION OF ORGANIC COMPOUNDS. I
}

\author{
BY V. VOORHEES WITH ROGER ADAMS ${ }^{1}$ \\ Received April 10, 1922
}

The use of colloidal platinum and palladium and of platinum and palladium black as catalysts, especially for the reduction of organic compounds, has become very general during the past 25 years. This interest has been due chiefly to the fact that many organic substances can conveniently be prepared only by such methods. The colloidal catalysts are in general somewhat more active and are more readily prepared, but are limited in their use due to the fact that only aqueous, dil. alcoholic or dil. acetic acid solutions may be employed and that difficulty may often arise in isolating the products. On the other hand, the use of platinum or palladium black is very convenient, since almost any solvent may be employed and the product isolated merely by filtering the platinum from the reaction solution and evaporation of the solvent. Of platinum and palladium, the former has been much more commonly used and is the one which will be discussed in this communication.

The method of preparation of platinum and palladium black, however, has not been given the study which it deserves. The earlier methods for making platinum black are very numerous and do not yield a product which is very active as a catalyst in the reduction of organic compounds.

0 . Loew ${ }^{2}$ first described the preparation of a satisfactory catalyst for organic reductions and his method, except for slight changes by other investigators, is the one which is used almost exclusively at the present time. Loew's method consists in the treatment of an aqueous chloroplatinic acid solution with formaldehyde and then precipitation of platinum black by the addition of alkali. The product is filtered, dried in vacuo and exposed to air or oxygen before being used. His directions have been changed, chiefly in regard to the temperature of precipitation and the nature of the alkali, first by Willstaetter and Hart; ${ }^{3}$ again, by Willstaetter and Waldschmidt-Leitz; and still again by Feulgen. ${ }^{5}$ These changes from the original directions of Loew result in the production of a more effective catalyst and eliminate a few of the experimental difficulties involved, such as that of filtration of the platinum black. Moreover, it is reported that the platinum produced by the modified directions is more uniform in its activity as a catalyst. Even with these improvements, the production of a very active catalyst cannot be relied upon unless the directions are followed with exactness. Drying in a good vacuum for 24 to 48 hours is very necessary if a high activity is to be obtained.

1 This paper is an abstract of a thesis by $V$. Voorhees presented in partial fulfilment of the requirements for the degree of Master of Science in chemistry at the University of Illinois.

2 Loew, Ber, 23, 289 (1900).

${ }^{3}$ Willstaetter and Hart, ibid., 45, 1472 (1912).

4 Willstaetter and Waldschmidt-Leitz, ibid, 54, 113 (1921).

s Feulgen, ibid., 54, 360 (1921). 
In this laboratory where catalytic reductions have been studied for the past few years, it has often been found that various students, using the same directions for making the catalyst, do not obtain a platinum of like activity. The investigation which is described here was carried out in order to determine whether a simpler and more reliable method could be found for the preparation of a highly active platinum catalyst.

$A$ brief description of the characteristics of a catalytically active platinum black is given below, since these observations led to the experiments and results which are now being reported. The presence of oxygen in platinum black is necessary for its activity as a catalyst in the hydrogenation of organic compounds. This has been noted by numerous investigators ${ }^{6}$ and tested quantitatively by Willstaetter and Waldschmidtl.eitz. ${ }^{7}$ In fact, the activity of platinum black seems to depend almost entirely upon the amount of oxygen absorbed. Willstaetter believes that the presence of oxygen is also necessary for catalysis with colloidal platinum, but recent work by Skita $^{8}$ has shown quite conclusively that this is not the case.

The form in which absorbed oxygen is present in the platinum black has been a question of great interest to chemists. Wöhler ${ }^{9}$ has shown convincingly that when platinum black absorbs oxygen there are formed true oxides or hydroxides of platinum. These consist for the most part of platinous oxide or, in the presence of water, platinous hydroxide, and a certain amount of platinic dioxide and its hydrate. From his experiments it is indicated that the platinous oxide or hydroxide is the active catalyst, or at least is an intermediate product in the formation of the active catalyst. This conclusion was drawn after a very careful study of platinum black which had absorbed oxygen and a comparison of its properties with those of the various oxides of platinum. Platinum black prepared by the usual method shows by its activity that it contains about 10 to $15 \%$ of platinous oxide and a few per cent. more of the higher oxides of platinum. The reactivity of the platinum ceases immediately as soon as the oxygen present has disappeared. It is well known that in catalytic reductions the oxygen in the platinum is not immediately removed by the hydrogen but reacts only gradually during a considerable period of time. When the oxygen in the platinum does become very small in amount the

${ }^{6}$ Öfversikt Finska Vetenskaps-Soc. Förh., 57, 267 (1900); Z. angew. Chem., 39, 24 (1904); Gazz. chim. ital., 34, 57 (1904); Ber., 51, 767 (1918); Compt. rend., 158, 409 (1914); Ber., 51, 768 (1918); Akad. Wetenschappen Amsterdam Proc, 29, 424 (1917).

${ }^{7}$ Ref. 4. In this paper an excellent review is given of the various papers on the mechanism by which the oxygen activates the platinum as a catalyst. See also Ber. 55, 073 (1922).

${ }^{8}$ Skita, ibid., 55, 139 (1922).

- Wöhler, ibid., 36, 3475 (1906). 
reduction slows down or stops altogether. It is merely necessary, however, to agitate the platinum with oxygen or air in order to revivify it.

The state of division of a platinum catalyst is also important and recent modifications in the preparation of platinum black have had as one of their objects the formation of a more finely divided product. It is most probable that the state of division and the absorption of oxygen go hand in hand.

From these properties of platinum black it was concluded that the oxides of platinum, if prepared in a finely divided state, would be as effective catalysts in hydrogenation as any which could possibly be produced. They would be distinctly more active than the best platinum black since the maximum amount of oxygen in the latter as platinum oxides, is at the most only about $20 \%$. Provided the oxide is finely divided, it would probably make little difference what particular oxide of platinum is used, since the hydrogen would reduce the higher oxides to the lower oxide, presumably the active catalyst. If these oxides are usable, even though they may be merely as active as ordinary platinum black, they would have a distinct advantage over it becatuse of the ease with which many of them may be prepared. For example, certain tedious details such as the careful drying of platinum black could be eliminated. Also the oxides of platinum, since the oxygen is present, could be used when wet, while platinum black precipitated from solution must be thoroughly dried and exposed to the air for the absorption of oxygen before it becomes most active as a catalyst. Experiments fully substantiate the predictions: many of the oxides of platinum are more effective as catalysts than platinum black made by the usual method.

All of the various oxides of platinum described in the literature are now being studied as catalysts. Of these, platinous hydroxide formed as described by the method of Thompsen ${ }^{10}$ has been made already. This compound is a catalyst and about as active as ordinary platinum black. The necessary conditions of precipitation and the physical characteristics of the product, however, are not ideal and this probably accounts for its lack of great activity. It seemed likely that a proper fusion method might be more suitable for the formation of a finely divided oxide which might be easily handled. Yorgensen ${ }^{11}$ has already described a poorly defined oxide of platinum produced by the fusion of sodium chloroplatinate with sodium carbonate. Such a method would hardly be expected th produce an active catalyst since the temperature of such a fusion mixtur. must reach $850^{\circ}$ or over. Such high temperatures are known to interfere often with the activity of a catalyst. Moreover, it has been shown by Wöhler ${ }^{9}$ that both platinous oxide and platinic dioxide decompose fairly

10 Thompsen, J. prakt. Chem., [2] 15, 294 (1876).

$"$ Yorgensen, ibid., [2] 16, 344 (1877). 
rapidly when held for any length of time at a temperature of $450^{\circ}$. The most appropriate method conceivable for the formation of an oxide of platinum is the one used so often for the oxides of other metals, namely, the fusion of the nitrate. ${ }^{12}$ In the case of platinum, this may be carried out by fusing chloroplatinic acid and sodium nitrate. Since sodium nitrate fuses at $312^{\circ}$, ideal conditions are obtainable. It is actually found that an extremely active oxide of platinum is thus produced and this is now being compared with platinum black and with oxides made in various ways. Preliminary results indicate that this is a higher oxide of platinum, possibly platinum trioxide. A more detailed description of this substance and its chemical properties will be given in a communication that will appear soon.

When the ordinary commercial chloroplatinic acid crystals are treated with an excess of sodium nitrate and fused, an evolution of nitrogen dioxide takes place and a precipitate forms in the melt. Upon cooling and washing with water, the new oxide of platinum is obtained. It is brown in color but the shade varies with the change in conditions during fusion. The whole procedure is extremely simple and within half an hour a large amount of catalyst can be prepared for use.

The formation of this oxide of platinum is not limited to a fusion of chloroplatinic acid with sodium nitrate, but may be produced by the fusion with nitrates of other metals. Moreover, low-melting salts of certain other acids may be used with success. The amount of the salt used for the fusion and the temperature employed have a decided effect upon the nature of the catalyst. All of these conditions are now being studied quantitatively and will be reported later.

The new catalyst seems to be less sensitive toward poisons than ordinary platinum black. It has, however, the same disadvantage as platinum black when used for the reduction of readily oxidizable compounds. Much of the oxygen in the catalyst is probably removed, due to the oxidation of the substance to be reduced. Platinum is formed which is only slightly reactive until shaken again with air or oxygen. The solvent used for the substance to be reduced affects the speed of reduction.

The catalyst has already been used for the reduction of phenol and certain substituted phenols to hexahydrophenols, for certain pyridine detivatives to piperidine derivatives, of vanillin and salicyl aldehyde to the corresponding alcohols, of aromatic nitro compounds to amines, of methyl-

${ }^{12}$ No one has attempted to prepare an oxide of platinum by such a method. The nearest approach lies in the work of Davy, who found that platinum sulfide dissolves in fuming nitric acid and that when this solution is evaporated to dryness and the residue treated with alcohol, platinum black is produced (Phil. Mag., 1820, 330). Hittorf also found that a brown oxide of platinum is formed by electrolytic oxidation of platinum when platinum strips are used as electrodes in a bath of fused potassium or sodium nitrate (Pharm. Zentr., 1848, 23). 
ethyl ketone to sec. butyl alcohol, and of certain heterocyclic compounds to the corresponding saturated derivatives. From these results, it may be concluded that this catalyst will probably work for all cases where platinum black can be used. All of these reductions mentioned were carried out with hydrogen under a pressure of 1.5 to $3 \mathrm{~atm}$., but it is inconceivable that this has any more effect with this catalyst than with ordinary platinum black.

The method of procedure for the reduction of compounds consists, in general, of dissolving in a suitable solvent the compound to be reduced, adding the catalyst, removing the air, and then treating with hydrogen. As soon as the hydrogen is introduced and the reaction mixture is shaken, the brown catalyst becomes black, due, presumably, to the reduction of the higher oxide to a lower one. The time necessary for this change of the catalyst depends to a considerable extent upon the way in which the catalyst has been formed and on the nature of the solvent used. For the catalyst, the preparation of which is described in this paper, the time necessary varies from a few seconds to 15 or 20 minutes. As soon as it becomes black it either becomes spongy in appearance or takes on a finely divided form which readily remains suspended in the reaction mixture. The platinum which is now present is essentially the same as a very active platinum black made in the usual way and is similar in all its properties. In many cases the reduction will take place so rapidly that the reaction mixture will actually become hot, but the speed of reduction depends upon the nature of the compound which is being studied. When the reduction is complete, the suspended platinum can be made to settle merely by shaking the reaction mixture with air for a short time, after which it may be readily filtered off or the solution may be decanted. After an amount of catalyst has reduced so much of the unsaturated compound that it has lost the greater part of its activity, it may be revivified by shaking a short time with air just as is customary when using platinum black.

It is obvious that the oxide of platinum prepared as described is not a catalyst in itself, but is converted by the hydrogen into the lower oxide which presumably is the active catalyst. In the study of the various oxides of platinum there is, therefore, a third condition which must be observed in the preparation, namely, that a product is formed which is readily reduced by hydrogen to the active catalyst. It was observed that when the sodium nitrate and chloroplatinic acid are fused at a temperature of $700^{\circ}$ or higher, an oxide is formed which catalyzes the reduction of organic substances only slowly; moreover, in certain instances, from 1 to 2 hours is required before the brown oxide turns black and becomes active as a catalyst.

The oxides of palladium and iridium are formed in a similar manner to 
the oxide of platinum, and an investigation of these products as catalysts which has shown them to be very active, is now being continued.

\section{Experimental}

Preparation of the Oxide of Platinum.-In a $150 \mathrm{cc}$. porcelain casserole or Pyrex beaker, is prepared a solution of crystals of chloroplatinic acid corresponding to $1 \mathrm{~g}$. of platinum in $5 \mathrm{cc}$. of water. To this is added $20 \mathrm{~g}$. of sodium nitrate. The mixture is heated gently over a Bunsen flame and stirred with a glass rod while the water is expelled. The mixture is then heated until complete fusion takes place, which requires a temperature of about $300^{\circ}$. Brown oxides of nitrogen are evolved at about $320^{\circ}$ and a brownish powder precipitates in the fusion mixture. Il is advisable to continue the stirring during the fusion in order to prerent spattering and to prevent caking of the precipitate on the bottom of the casserole. The melt is kept hot until the copious evolution of nitrogen oxides has almost ceased, a process which requires from 5 to 15 minutes, depending upon the temperature at which the fusion mixture is held. This point is easily observed, however, as there is a sudden derrease in amount of gases given off, although the brown fumes never rease completely even after the precipitation of the oxide seems to be complete. The mass is now allowed to cool and then treated with $50 \mathrm{cc}$. of water. The brown precipitate settles to the bottom and can be washed by decantation once or twice and then washed on a filter until free from nitrates as shown by the absence of the ferrous sulfate test. This requires about 100 to $125 \mathrm{cc}$. of water. Toward the end of the washing there may be traces of oxide that tend to be carried through. It is desirable, therefore, to keep the filtrates. The filtration and washing are readily completed in 15 to 20 minutes. The product that is thus obtained may either be used directly or may be dried in a desiccator and kept until needed.

Discussion of Reaction.-In studying the best conditions under which the fusion may be carried out, a platinum thermocouple was used for determining the temperature of the fusion mixture. It was observed that the higher the temperature used during the fusion, the more complete was the precipitation of the platinum. On the other hand, the product formed is not as active a catalyst as that produced at lower temperatures. It seems advisable to use a temperature about $450^{\circ}$ if the best results are to be obtained. At $600^{\circ}$ and $700^{\circ}$ the product seems to be slightly more yellow in color and causes the reduction of various organic compounds to take place much more slowly. It is quite unnecessary, however, to record the temperature in the preparation. A small flame just sufficient to cause the fusion to take place as mentioned, will invariably give very satisfactory results. 
The filtrate, after the separation of the catalyst from the fusion mixture, should be evaporated to dryness. The small amount of platinum which always remains may then be precipitated quantitatively by adding a few grams of sodium carbonate and fusing at a comparatively high temperature. The melt is then cooled and the platinum recovered by washing free from the salts.

It is advisable always to test for platinum in all the filtrates before cliscarding any of them since there is a tendency for a small amount of the platinum to remain in solution. A very satisfactory and delicate test for platinum, described by Wöhler, ${ }^{13}$ consists in making the solution acid with hydrochloric acid and adding a few drops of stannous chloride. A yellow color develops when platinum is present and a brown color when it is in large amount. If any doubt exists as to whether the solution is yellow, it should be shaken with a small amount of ether, under which conditions the yellow color concentrates in the upper layer, thus indicating the presence of platinum.

The oxide may be dried rapidly by washing several times with alcohol. If this is carried out, however, care must be taken to prevent exposure to the air since spontaneous combustion will take place.

If organic matter is present in the chloroplatinic acid such as is generally the case when recovered platinum is used, it will be oxidized during the fusion and seems to have no deleterious effect.

The oxide may be produced by using a much smaller proportion of sodium nitrate to chloroplatinic acid than that suggested. With the larger quantity, however, filtration of the oxide takes place more readily with practically no tendency to become colloidal and pass through the filter.

There always remains in the casserole or beaker a thin film of oxide which cannot be removed by the ordinary means. This is dissolved best by treating with a little constant-boiling hydrobromic acid, which immediately dissolves it. The solution thus obtained may then be added to the next fusion mixture.

There is always a certain amount of the oxide which remains sticking to the filter paper during filtration, so that these papers should be ignited and the residue added to any platinum catalyst which has already become inactive and requires redissolving and reprecipitation.

Apparatus.-The general form of apparatus employed in the reduction experiments is that described by Skita $^{14}$ and modified by Lochte, Noyes and Bailey. ${ }^{15}$ As a container for hydrogen a Prest-o-lite tank was used from which the filling had been removed. The top of the tank contains two openings. In one of these is welded a tube holding a gage and valve;

${ }^{13}$ Wöhler, Chem. Ztg., 31, 938 (1907).

14 Skita, Ber., 45, 3589 (1912).

${ }^{15}$ Lochte, Noyes and Bailey, This Journal, 43, 2601 (1921). 
through this tube the hydrogen from a large cylinder is introduced into the tank. In the second opening is welded another tube with two outlets, each of which holds a needle valve. These outlets are so arranged that it is possible to shut off the tank from either outlet. One is used for the vacuum and the second for a tube leading to the reaction-mixture bottle. The Prest-o-lite tank is so calibrated that merely by reading the fall in pressure and the temperature, the amount of hydrogen absorbed by the reaction mixture can be quite closely determined. The connection between the Prest-o-lite tank and hydrogenating bottle may be a copper tube connected to a heavy taped rubber tube which is in turn connected to a glass tube inserted through the stopper of the bottle. The rubber tube and stopper should be high grade and must be carefully boiled with alkali before being used.

Reduction Experiments.-It has been found that the speed of reduction of various organic compounds is approximately proportional to the amount of catalyst used. It therefore follows that in the experiments described, the speed can be greatly increased by using more catalyst.

In the preparation of vanillyl alcohol from vanillin, several amounts of material have been reduced in succession, with the same platinum. The reduction of successive amounts of the other substances has not been attempted, but would undoubtedly take place just as satisfactorily.

The experiments described below were carried out under an initial pressure of hydrogen of $2.75 \mathrm{~atm}$., so that an exact comparison can be made among the various experiments.

In each case, the reduction of about $95 \%$ of the material takes place rapidly; the speed of reduction then slows down. The reduction of the last $5 \%$ often requires almost as much time as is necessary for the reduction of the first $95 \%$ of material.

The products are isolated in every case by shaking with air, in order to cause the platinum to settle, filtering or decanting the solution. The product may then be either distilled in vacuo or crystallized, according to its physical properties.

Vanillin to Vanillyl Alcohol.-A solution is made of $20 \mathrm{~g}$. of vanillin in $100 \mathrm{cc}$. of alcohol and $0.5 \mathrm{~g}$. of catalyst is added. The reduction is complete in about 50 minutes after which the reaction mixture is shaken with air, the solution decanted and a second $20 \mathrm{~g}$. of vanillin in $100 \mathrm{cc}$. of alcohol added. The second reduction requires about 110 minutes, a third of a similar nature, 125 minutes, and a fourth about 120 minutes.

Ethylmethyl Ketone to sec. Butyl Alcohol.-Eighty g. of ethylmethyl ketone is treated with $0.5 \mathrm{~g}$. of catalyst. The reduction is complete in about 4 hours and practically a quantitative yield of product may be isolated.

Phenol to Cyclohexanol.-A solution is made of $62 \mathrm{~g}$. of phenol in $50 \mathrm{cc}$. of alcohol and $0.65 \mathrm{~g}$. of catalyst is added. The time necessary for complete reduction is 6.5 hours and a yield of $54 \mathrm{~g}$. of pure cyclohexanol is obtained. A trace of benzene can be detected by its odor. 
Salicyl Aldehyde to Saligenin.-A solution is made of $30 \mathrm{~g}$. of salicyl aldehyde in $60 \mathrm{cc}$. of alcohol and $0.25 \mathrm{~g}$. of catalyst is added. The reduction is complete in about 75 minutes and practically a quantitative yield is obtained.

Nicotinic Acid Hydrochloride to Nipocotinic Acid Hydrochloride.-A solution is made of $10 \mathrm{~g}$. of nicotinic acid hydrochloride in $150 \mathrm{cc}$. of water and $1.0 \mathrm{~g}$. of catalyst is added. The reduction is complete in about 2 hours. The product may be isolated by vacuum evaporation of the solution.

\section{Summary}

1. Since experiments indicate that the oxygen which must always be present in platinum black if it is to be an active catalyst, is present in the form of an oxide of platinum, it was concluded that the oxides of platinum should be excellent catalysts. These are being studied.

2. An oxide of platinum has been formed by the fusion of chloroplatinic acid with sodium nitrate and has proved to be an excellent catalyst.

3. Various types of organic compounds such as phenols, pyridine derivatives, aromatic and aliphatic aldehydes and heterocyclic compounds have been reduced. The speed of reduction with this catalyst is greater than when ordinary platinum black is used.

URBANA, ILLINOIS

\section{NOTE}

Vanillin Glyceride.-The attention of the writer was recently called to a deposit which had formed in a flavoring mixture consisting essentially of alcohol, glycerin, and vanillin. The precipitate was easily purified by filtration and washing with water and, thus isolated, was a white, almost odorless, micro-crystalline powder, slightly soluble in water and ether. It was moderately soluble in cold alcohol, easily on heating, separating from solution on cooling in distinct crystalline plates. It was also easily soluble in $0.5 \mathrm{~N}$ potassium hydroxide solution, without, however, showing any definite neutralization, and was precipitated in crystals on the addition of acid, behaving thus like a phenol.

On warming the crystals with water, they dissolved slowly and the odor of vanillin became apparent. After a few hours' heating, vanillin alone crystallized from the cooled solution, and in the mother liquor glycerin was found by the usual tests. The hydrolysis was greatly hastened by acid catalysts, and an approximate analysis of the compound was made as follows.

$2.0795 \mathrm{~g}$. of substance was mixed with $20 \mathrm{cc}$. of warm water, and $1 \mathrm{cc}$. of $0.5 \mathrm{~N}$ hydrochloric acid added. The crystals dissolved immediately, and vanillin separated. To insure a complete hydrolysis, the mixture was heated for 1 hour at $90-100^{\circ}$. On cooling, the solution was extracted 4 times with ether, in $10 \mathrm{cc}$. portions. The ether extract, evaporated to constant weight at $50^{\circ}$, was nearly pure vanillin; the aqueous solution similarly evaporated left a residue of slightly discolored glycerin.

Calc. for vanillin: ( $1 \mathrm{~mol}$ ) $67.25 \%$. Found: $1.405 \mathrm{~g}$, or $67.5 \%$.

Calc. for glycerin: (1 mol.) $40.71 \%$. Found: $0.818 \mathrm{~g}$, or $39.3 \%$. 